\title{
Small excitonic complexes in a disk-shaped quantum dot
}

\author{
Ricardo Pérez ${ }^{1,2}$ and Augusto Gonzalez ${ }^{10}$ \\ ${ }^{1}$ Centro de Matemáticas y Fúsica Teórica, Calle E No. 309, Ciudad Habana, Cuba \\ ${ }^{2}$ Universidad de Antioquia, A.A. 1226, Medellín, Colombia
}

(November 10, 2018)

\begin{abstract}
Confined excitonic complexes in two dimensions, consisting of $N_{e}$ electrons and $N_{h}$ holes, are studied by means of Bethe-Goldstone equations. Systems with up to twelve pairs, and asymmetric configurations with $N_{e} \neq N_{h}$ are considered. The weak confinement regime gives indication of weak binding or even unbinding in the triexciton and the four-exciton system, and binding in the higher complexes.
\end{abstract}

PACS numbers: 71.35.Ee, 78.65.-w

Keywords: quantum dots, electron-hole systems, Bethe-Goldstone equations

Typeset using REVTEX 


\section{INTRODUCTION}

Although the excitonic matter as a research object has already a relatively long history, dating back to pioneer theoretical ideas in the sixties [1], the study of small excitonic complexes confined in regions of nanometer scale became possible only recently, and is closely related to dramatic advances in semiconductor technology.

Due to the smallness of the exciton lifetime in semiconductors (hundreds of picoseconds, sometimes even greater), signatures of excitonic complexes are looked for mainly in optical experiments. Exciton and biexciton peaks in photoluminescence have been identified in quantum dots [2,3]. Recently, a confocal microscopy technique has been applied to resolve the luminescence coming from a single self-assembled quantum dot [⿴囗十 . The power of the excitation laser is such that up to six pairs are excited simultaneously in the dot. Clearly distinct lines coming from transitions between multiexcitonic complexes are identified.

The calculations presented in our paper, although unrealistic, are inspired by the experimental work [4]. We address a different question, i. e. the dependence of the ground-state energy on the number of pairs. From this dependence, the lowest optical absorption line related to the creation of an electron-hole pair in the background of $N$ photo-excited pairs may be extracted.

On the other hand, small excitonic systems have been widely studied theoretically in

order to describe the optical properties of bulk and low-dimensional semiconductors. To the best of our knowledge, one of the most complete studies is the paper [5], in which a variational approach is used to compute the properties of two- and tridimensional free (not confined) excitons with up to five particles. Our work has also a close connection with that paper.

By using the Bethe-Goldstone (BG) equation to compute the ground-state energies, we are able to rise the number of particles in the complex from a maximum of five in the variational approach [5] to 24. Asymmetric systems with $N_{e} \neq N_{h}$ are studied as well. In the weak confinement regime, we can also address the question about the stability of the free 
complexes. And in fact, our results give indications that the free triexciton and the fourexciton system are unstable (or weakly bound), whereas the higher complexes are stable. These properties have a distinct trace in the optical absorption, and may be confirmed by the experiments.

The present paper is complementary to [6], in which a BCS variational approach is used to study intermediate-size complexes, containing from 12 to 180 particles. For those sizes where both approaches overlap, as a rule BCS gives lower energy values for strong confinement, whereas BG gives better energies in the weak confinement regime.

\section{THE BETHE-GOLDSTONE EQUATION}

We will study a two-dimensional model of $N_{e}$ electrons and $N_{h}$ holes confined by a parabolic potential in the plane of motion, and interacting via pure Coulomb potentials. Only one conduction and one valence band, both ideally parabolic, will be considered, and the masses of holes and electrons will be supposed to be equal. By choosing the scales of distances and energies as $\sqrt{\hbar / m \omega_{0}}$ and $\hbar \omega_{0}$, we get the dimensionless Hamiltonian

$$
H=\sum_{i=1}^{N_{e}}\left(\frac{p_{i}^{2}}{2}+\frac{r_{i}^{2}}{2}\right)+\sum_{i=1}^{N_{h}}\left(\frac{p_{i}^{2}}{2}+\frac{r_{i}^{2}}{2}\right)+\beta\left[\sum_{i<j}^{N_{e}} \frac{1}{r_{i j}}+\sum_{i<j}^{N_{h}} \frac{1}{r_{i j}}-\sum_{i}^{N_{e}} \sum_{j}^{N_{h}} \frac{1}{r_{i j}}\right],
$$

where the $\overrightarrow{r_{i}}$ are in-plane coordinates for the particles, $r_{i j}=\left|\overrightarrow{r_{i}}-\overrightarrow{r_{j}}\right|, \omega_{0}$ is the frequency of

the confining potential, $\kappa$ is the dielectric constant of the semiconductor, $\beta=\sqrt{E_{c} /\left(\hbar \omega_{0}\right)}$, and $E_{c}=m e^{4} /\left(\kappa^{2} \hbar^{2}\right)$ is the characteristic Coulomb energy.

$\beta$ is the only parameter entering the Hamiltonian (11). It may be thought of as the inverse of the confinement strength. In the $\beta=0$ limit, we have a picture of non-interacting fermions which energy levels and one-particle quantum states are those of the $2 D$ harmonic oscillator. The electron (hole) states are grouped into shells with "magic numbers" $N_{e}, N_{h}=$ $2,6,12, \ldots$. As $\beta$ grows, correlations between particles become more and more important and the previous picture is modified.

One way to go beyond the independent-particle picture is the independent-pair approx- 
imation in which an exact treatment of the two-body correlations is made. Its main component is the Bethe-Goldstone (BG) equation, extensively used in nuclear matter and finite nucleus calculations [7,8].

The BG equation applies only to fermionic systems. It describes the motion of an independent pair of fermions in the system. The rest of the particles excerpt an indirect influence on the pair motion through the Pauli principle. The equation takes the form:

$$
\left(T_{1}+T_{2}+Q_{\alpha \gamma} V\right) \psi_{\alpha \gamma}=E_{\alpha \gamma} \psi_{\alpha \gamma}
$$

where $\alpha$ and $\gamma$ label the states of each fermion in the pair. These states are below the Fermi level. The $T_{i}$ are the one-particle terms in the Hamiltonian, $V$ is the two-body interaction potential (Coulomb), and $Q_{\alpha \gamma}$ is a projection operator given by

$$
\left.Q_{\alpha \gamma}=\mid \alpha \gamma\right)\left(\alpha \gamma\left|+\sum_{\mu^{\prime}, \lambda^{\prime}}\right| \mu^{\prime} \lambda^{\prime}\right)\left(\mu^{\prime} \lambda^{\prime} \mid\right.
$$

where the sum runs over states above the Fermi level. $Q$ projects a given function onto states over the Fermi level. $\left(\overrightarrow{r_{1}} \overrightarrow{r_{2}} \mid \alpha \gamma\right)$ is the unsymmetrized product of two non-interacting one-particle eigenfunctions (we use the notation given in [9]). $E_{\alpha \gamma}$ is the pair energy, and $\psi_{\alpha \gamma}-$ its wave function.

Equation (2) is formally similar to a pair scattering equation, except for the presence of the projection operator (and the fact that all of the states in the external quadratic potential are bound states). The pair wave function $\psi_{\alpha \gamma}$ is looked for in the form

$$
\left.\left.\psi_{\alpha \gamma}=\mid \alpha \gamma\right)+\sum_{\mu^{\prime}, \lambda^{\prime}} C_{\mu^{\prime} \lambda^{\prime}}^{\alpha \gamma} \mid \mu^{\prime} \lambda^{\prime}\right)
$$

and the total energy is computed from

$$
E=\sum_{\alpha} \epsilon_{\alpha}^{(0)}+\sum_{\alpha<\gamma} \epsilon_{\alpha \gamma}
$$

where $\epsilon_{\alpha \gamma}=E_{\alpha \gamma}-\epsilon_{\alpha}^{(0)}-\epsilon_{\gamma}^{(0)}$. The corrections coming from the BG equations are proven to be equivalent to summing up all the ladder diagrams in the linked-cluster expansion for the energy [10]. 
Multiplying (2) from the left by $(\mu \lambda \mid$ or $(\alpha \gamma \mid$, we get

$$
\begin{array}{r}
\left(\epsilon_{\mu}^{(0)}+\epsilon_{\lambda}^{(0)}-E_{\alpha \gamma}\right) C_{\mu \lambda}^{\alpha \gamma}+\sum_{\mu^{\prime}, \lambda^{\prime}}\left(\mu \lambda|V| \mu^{\prime} \lambda^{\prime}\right) C_{\mu^{\prime} \lambda^{\prime}}^{\alpha \gamma}=-(\mu \lambda|V| \alpha \gamma) \\
E_{\alpha \gamma}=\epsilon_{\alpha}^{(0)}+\epsilon_{\gamma}^{(0)}+(\alpha \gamma|V| \alpha \gamma)+\sum_{\mu^{\prime}, \lambda^{\prime}}\left(\alpha \gamma|V| \mu^{\prime} \lambda^{\prime}\right) C_{\mu^{\prime} \lambda^{\prime}}^{\alpha \gamma}
\end{array}
$$

Equation (6) may be seen as a linear system of equations for the coefficients $C_{\mu \lambda}^{\alpha \gamma}$, from which we obtain $C_{\mu \lambda}^{\alpha \gamma}=C_{\mu \lambda}^{\alpha \gamma}\left(E_{\alpha \gamma}\right)$. Then, the transcendental equation (ך) is solved for $E_{\alpha \gamma}$.

One shall notice that the Coulomb interaction does not change neither the angular momentum of the pair nor the spin of the particles. The matrix elements are real and have the following properties:

$$
(\alpha \gamma|V| \mu \lambda)=(\gamma \alpha|V| \lambda \mu)=(\mu \lambda|V| \alpha \gamma)
$$

Due to these properties, the matrix entering the linear system is symmetric.

In order to solve the BG equation we have to identify, given an initial pair, all the possible final states above the Fermi level preserving angular momentum and spin. Then we solve the linear system and the transcendental equation to get the pair energy. One shall take into account that there are three kinds of pairs in the system, namely e-e, h-h and e-h. In the second term of (5), $\alpha<\gamma$ means that for identical particles (e-e and h-h) a state $\mid \alpha \gamma$ )

should be counted only once. For e-h pairs, however, we should take into account the two possibilities, i. e. $\left(\alpha_{e} \gamma_{h}\right)$ and $\left.\mid \alpha_{h} \gamma_{e}\right)$.

In our calculations, up to 272 harmonic-oscillator one-particle states (16 shells) are included. Whenever possible, the e-h, spatial inversion and time-reversal symmetries are used to reduce the actual number of equations to solve. For example, in the $N_{e}=N_{h}=6$ problem, 15 systems of linear equations with roughly 700 unknowns each, and 15 nonlinear equations for the pair energies are solved.

\section{SYMMETRIC $\left(N_{E}=N_{H}\right)$ SYSTEMS}

In this section we present the results obtained for symmetric systems, where $N_{e}=N_{h}=$ $N$. The parameter $\beta$ is varied in the interval $(0,2.5)$. 
The first step in our Bethe-Goldstone calculation is to define filled and empty levels, i. e. the Fermi surface. To this end, a Hartree-Fock calculation was implemented. The harmonic-oscillator states were filled in accordance with the Hartree-Fock results. We show them in spectroscopic (nuclear) notation in Table [1. $2 p_{-}$, for example, means the second level with $l_{z}=-1$. $S_{e}$, and $S_{h}$ refer to the total electron and hole spins respectively, and $L$ is the total angular momentum (along the $z$-axis).

\section{A. The biexciton}

For the biexciton, our starting configuration is one in which the first harmonic oscillator shell is filled for both electron and holes (see Table [I). This state has zero total angular momentum and total spin. In order to write down the Bethe-Goldstone equations we shall identify all possible pairs below the Fermi level. In the e-e and h-h sectors there is only one pair, but we have 4 e-h pairs. For a given initial pair, the number of final pair states above the Fermi level depends on the number of harmonic oscillator shells, $N_{\text {shell }}$, included in the

calculations. Figure 1 shows the results for the energy as a function of $\beta$ and $N_{\text {shell }}$. The solid line is a two-point Padé approximant, which construction is described below.

A few remarks shall be given at this point. First, notice that the convergence is slow. Second, the BG results do not exactly reproduce the perturbative energies at small values of $\beta$. It means simply that the characteristic distances are much smaller than the characteristic pair dimensions, and thus the independent pair approximation breaks down. Fortunately, in this strong-confinement regime the energy is dominated by the one-particle energies, which are properly accounted for by the BG approach. Finally, the BG energies are seem to overestimate the actual binding energies at large $\beta$.

The two-point Padé approximant for the ground-state energy is constructed in the following way [11,12]. The asymptotic expansions

$$
\left.E\right|_{\beta \rightarrow 0}=b_{0}+b_{1} \beta+b_{2} \beta^{2}+\mathcal{O}\left(\beta^{3}\right)
$$




$$
\left.E\right|_{\beta \rightarrow \infty}=a_{0} \beta^{2}+a_{2}+\mathcal{O}\left(1 / \beta^{2}\right),
$$

are used, where $b_{0}=4, b_{1}=-2.50662, b_{2}=-2.92$ [6], and $a_{0}=-2.1928$ [0], $a_{2}=1 . a_{0}$ is the ground-state energy of the free biexciton in two dimensions, and $a_{2}$ comes from the center of mass oscillation, contributing with a one to the ground-state energy.

The two-point approximant is a rational function interpolating between (9) and (10):

$$
P(\beta)=b_{0}+b_{1} \beta+\frac{b_{2} \beta^{2}+p_{3} \beta^{3}+p_{4} \beta^{4}}{1+q_{1} \beta+q_{2} \beta^{2}} .
$$

The values of the parameters are the following, $p_{3}=0.525372, p_{4}=-2.06239, q_{1}=0.83554$ and $q_{2}=0.940527$.

\section{B. $\mathrm{N}=3,4,5,6$ and 12}

Next, we present results for $N=3,4,5,6$ and 12 , computed with $N_{\text {shell }}=16$. The occupied harmonic-oscillator states are indicated in Table $\llbracket$. Note that, in some cases, the Hartree-Fock approximation does not suggest a unique state, thus we performed BG calculations with different occupations, and select the one corresponding to the minimal energy. For example, in the $N=3$ system we performed calculations also for a configuration very similar to the one given in Table [, but with total momentum $\mathrm{L}=2$.

The results are drawn in Fig. 2 in a "scaled" form, i. e. $E_{g s} / N^{3 / 2}$ vs. $\beta / N^{1 / 4}$. This scaling comes from the dependence $b_{0} \approx 4 / 3 N^{3 / 2}, b_{1} \approx-0.96 N^{5 / 4}, b_{2} \approx-1.65 N$ and $a_{0} \approx-N$ for large $N$ [6], but it is nicely satisfied even for the smallest systems. In this scaled drawing, the $N=4$ cluster clearly distinguishes as the less bound one.

The results at the largest values of $\beta$, i. e. in the weak confinement regime, can be taken as indications of stability or instability of the free clusters. The triexciton seems to be unbound (like in three dimensions [13]), and the four-exciton system - evidently unbound. However, the larger clusters are likely to be stable. The situation may be analogous to nuclei, where there is a small instability island around atomic number five. 
The differences $E_{N+1}-E_{N}$ are shown in Fig. 3. We notice that these differences enter the expression

$$
h \nu=E_{g a p}+E_{z}^{e}+E_{z}^{h}+E_{N+1}-E_{N},
$$

for the frequency of light creating a new electron-hole pair in the background of $N$ pairs. $E_{\text {gap }}$ is the semiconductor gap, and $E_{z}^{e, h}$ are the electron (hole) confinement energies in the $z$-direction.

As following from Fig. 3, the absorption peak corresponding to (12) will exhibit an interesting behaviour as a function of the number of photoexcited pairs, $N$. High values of $N$ can be reached by rising the laser excitation power. When $N$ is around four, we shall observe a highly blueshifted line, followed by a redshifted one as the power is further increased. The conclusions coming from our oversimplified model have only a qualitative predictive power. The effect is so pronounced, however, that we expect it may be observed in experiments.

\section{ASYMMETRIC CASES}

We also studied non-symmetric or non-neutral systems, in which $N_{e} \neq N_{h}$. In particular, the cases $N_{e}=4, N_{h}=2$ and $N_{e}=6, N_{h}=2$. The results for their ground state energies are shown in Fig. 4. In a non-neutral system, the energy is an increasing function of $\beta$ for low $\beta$ values, as for electrons [14], but for weaker confinement the $-\beta^{2}$ biexcitonic contribution dominates over the $\beta^{2 / 3}$ repulsion. A biexciton plus remaining electrons shall be seen at very large $\beta$. The $N_{e}=6, N_{h}=2$ system clearly reveals the change in slope.

\section{CONCLUSIONS}

In the present paper, we have shown that the Bethe-Goldstone equations may be used as a powerful method to study small confined excitonic clusters. Pure electronic quantum dots may be studied as well, and external electric and magnetic fields can be easily included 
in the calculations. Larger unbalanced systems with only one exciton may be studied to obtain the optical absorption and photoluminescence of small electronic quantum dots.

Our numerical results suggest a small instability island for the free clusters around $N_{e}=$ $N_{h}=4$. We showed that this instability causes the appearance of distinct absorption peaks as the laser excitation power is raised.

More realistic calculations, closely related to the experimental results [4], are in progress.

\section{ACKNOWLEDGMENTS}

The authors acknowledge support from the Universidad de Antioquia, Medellin. Part

of this work was done during a visit to the Abdus Salam ICTP under the Associateship Scheme and the Visiting Young Student Programme. Useful discussions with E. Lipparini are also acknowledged. 


\section{REFERENCES}

- $\quad$ Electronic mail: rperez@cidet.icmf.inf.cu

* $\quad$ Electronic mail: agonzale@cidet.icmf.inf.cu

[1] L. V. Keldysh in Bose-Einstein condensation, eds. A. Griffin, D. W. Snoke and S. Stringari, Cambridge Univ. Press (1995).

[2] J. Y. Marzin J. M. Gerard, A. Izrael, D. Barrier and G. Bastard, Phys. Rev. Lett. 73, 716 (1994); H. Drexler, D. Leonard, W. Hansen, J. P. Kotthaus and P. M. Petroff, Phys. Rev. Lett. 73, 2252 (1994).

[3] A. Kuther, M. Bayer, A. Forchel, A. Gorbunov, V. B. Timofeev, F. Schafer and J. P. Reithmaier, Phys. Rev. B 58, 7508 (1998).

[4] E. Dekel, D. Gershoni, E. Ehrenfreund, D. Spektor, J. M. Garcia and P. M. Petroff, Phys. Rev. Lett. 80, 4991 (1998).

[5] J. Usukura, Y. Suzuki and K. Varga, Phys. Rev. B 59, 5652 (1999).

[6] B. A. Rodriguez, A. Gonzalez, L. Quiroga, R. Capote and F. J. Rodriguez, Int. J. Mod. Phys. B (1999), to appear.

[7] H. A. Bethe, Annu. Rev. Nucl. Sci. 21, 93 (1971).

[8] A. de Shalit and H. Feshbach, Theoretical Nuclear Physics, Vol. 1, John Wiley \& Sons, New York (1974).

[9] J. P. Blaizot and G. Ripka, Quantum theory of finite systems, MIT Press, Cambridge (1986).

[10] B. D. Day, Rev. Mod. Phys. 39, 719 (1967).

[11] A. H. MacDonald and D. S. Ritchie, Phys. Rev. B 33, 8336 (1986).

[12] A. Gonzalez, J. Phys.: Condens. Matter 9, 4643 (1997). 
[13] K. Varga and Y. Suzuki, Phys. Rev. A 53, 1907 (1996).

[14] A. Gonzalez, B. Partoens and F. M. Peeters, Phys. Rev. B 56, 15740 (1997). 


\section{FIGURES}

FIG. 1. Convergence of the biexciton energy as a function of the number of harmonic-oscillator shells included in the calculations.

FIG. 2. Energies of the N-exciton systems as a function of $\beta$.

FIG. 3. Energy differences $E_{N+1}-E_{N}$ for the smallest clusters studied.

FIG. 4. Energies of the $N_{e}=4, N_{h}=2$, and $N_{e}=6, N_{h}=2$ systems. 


\section{TABLES}

TABLE I. Occupied electron and hole states for the BG calculations.

\begin{tabular}{|c|c|c|c|c|c|c|}
\hline$N_{e}$ & $N_{h}$ & electrons & holes & $S_{e}$ & $S_{h}$ & $\mathrm{~L}$ \\
\hline 2 & 2 & $1 s^{2}$ & $1 s^{2}$ & 0 & 0 & 0 \\
\hline 3 & 3 & $1 s^{2} 1 p_{+}^{1}$ & $1 s^{2} 1 p_{-}^{1}$ & $1 / 2$ & $1 / 2$ & 0 \\
\hline 4 & 4 & $1 s^{2} 1 p_{+}^{1} 1 p_{-}^{1}$ & $1 s^{2} 1 p_{+}^{1} 1 p_{-}^{1}$ & 1 & 1 & 0 \\
\hline 5 & 5 & $1 s^{2} 1 p_{+}^{2} 1 p_{-}^{1}$ & $1 s^{2} 1 p_{+}^{1} 1 p_{-}^{2}$ & $1 / 2$ & $1 / 2$ & 0 \\
\hline 6 & 6 & $1 s^{2} 1 p_{+}^{2} 1 p_{-}^{2}$ & $1 s^{2} 1 p_{+}^{2} 1 p_{-}^{2}$ & 0 & 0 & 0 \\
\hline 12 & 12 & $1 s^{2} 1 p_{+}^{2} 1 p_{-}^{2} 2 s^{2} 1 d_{+}^{2} 1 d_{-}^{2}$ & $1 s^{2} 1 p_{+}^{2} 1 p_{-}^{2} 2 s^{2} 1 d_{+}^{2} 1 d_{-}^{2}$ & 0 & 0 & 0 \\
\hline 4 & 2 & $1 s^{2} 1 p_{+}^{1} 1 p_{-}^{1}$ & $1 s^{2}$ & 1 & 0 & 0 \\
\hline 6 & 2 & $1 s^{2} 1 p_{+}^{2} 1 p_{-}^{2}$ & $1 s^{2}$ & 0 & 0 & 0 \\
\hline
\end{tabular}




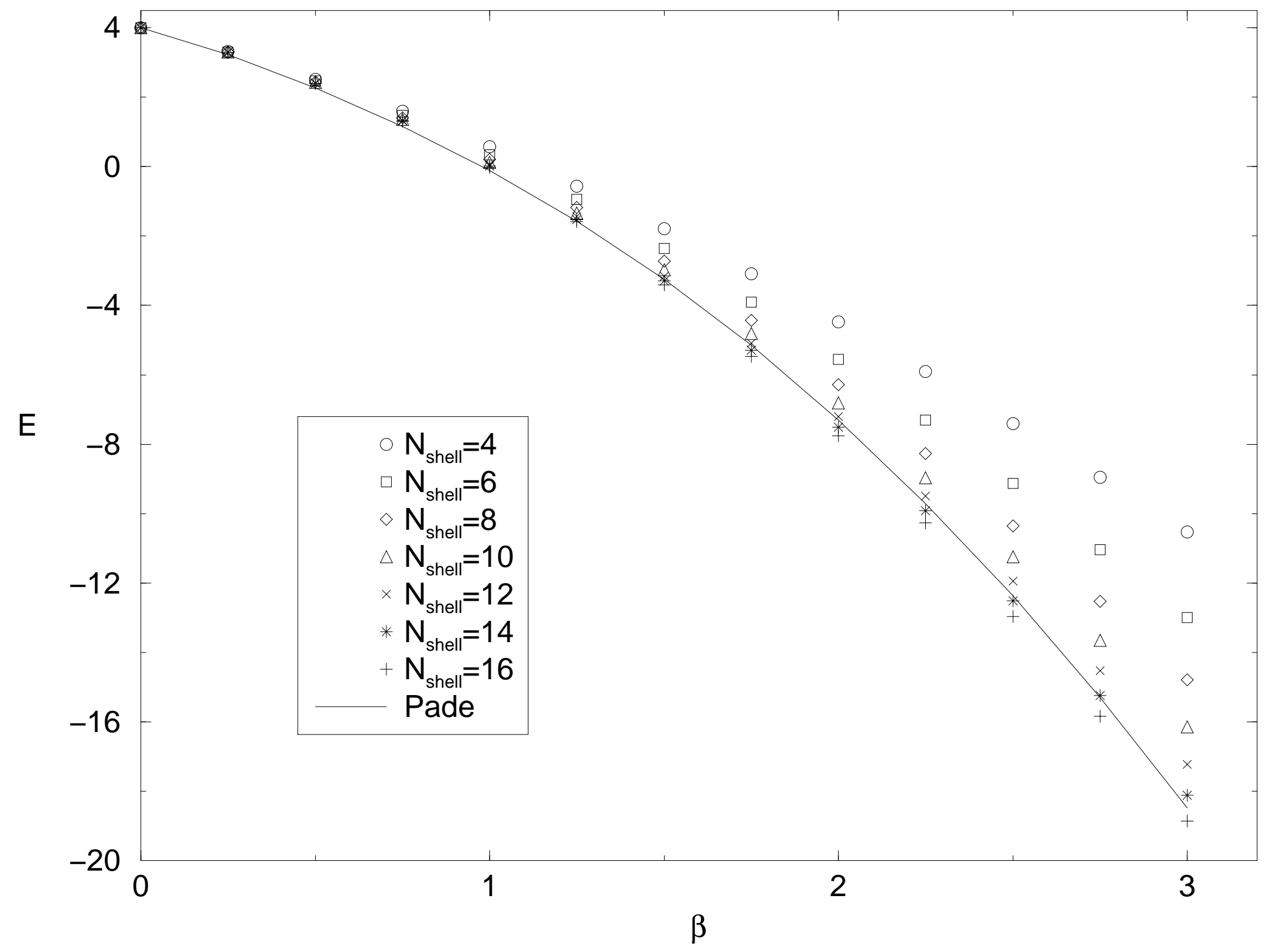




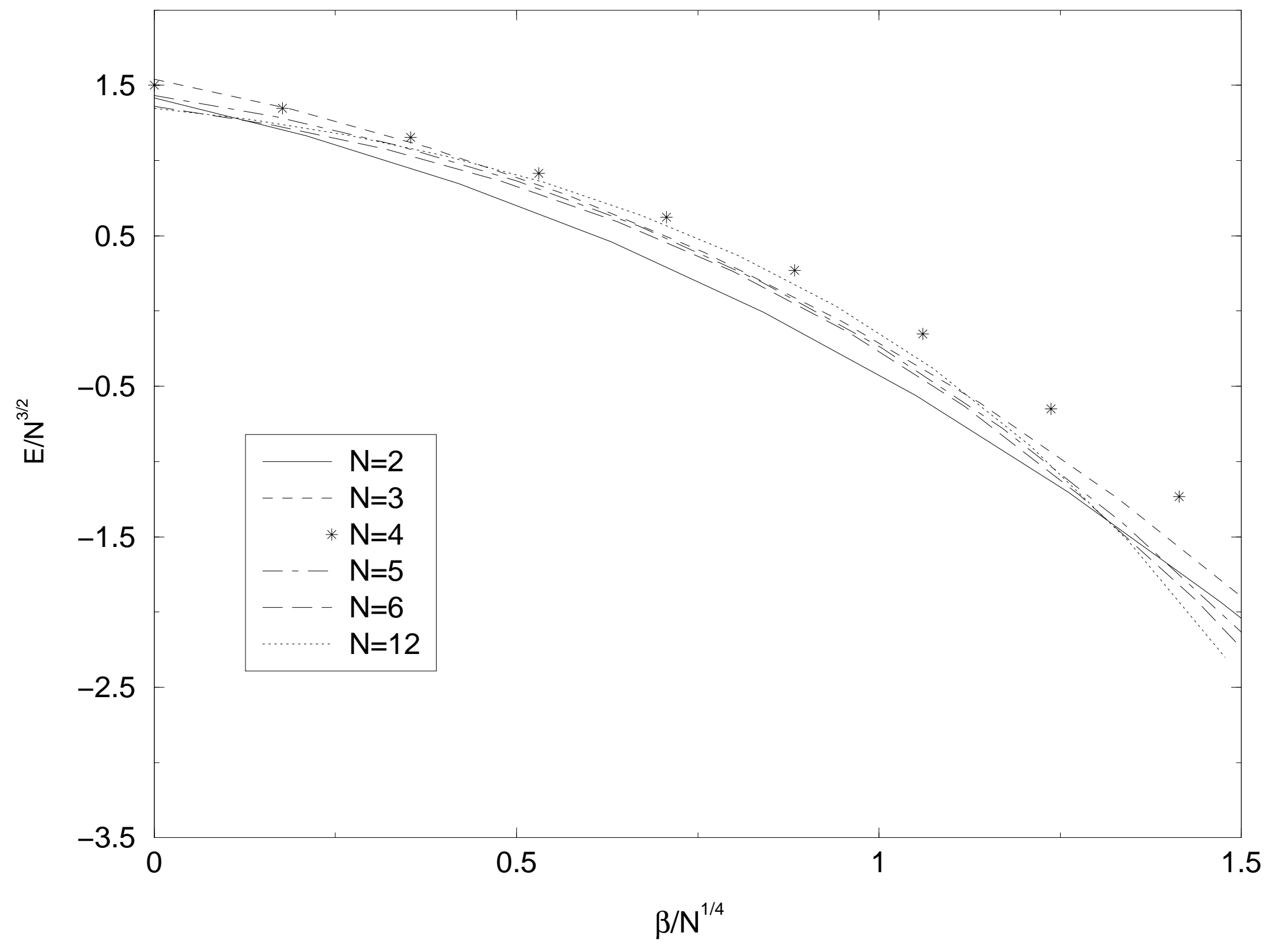




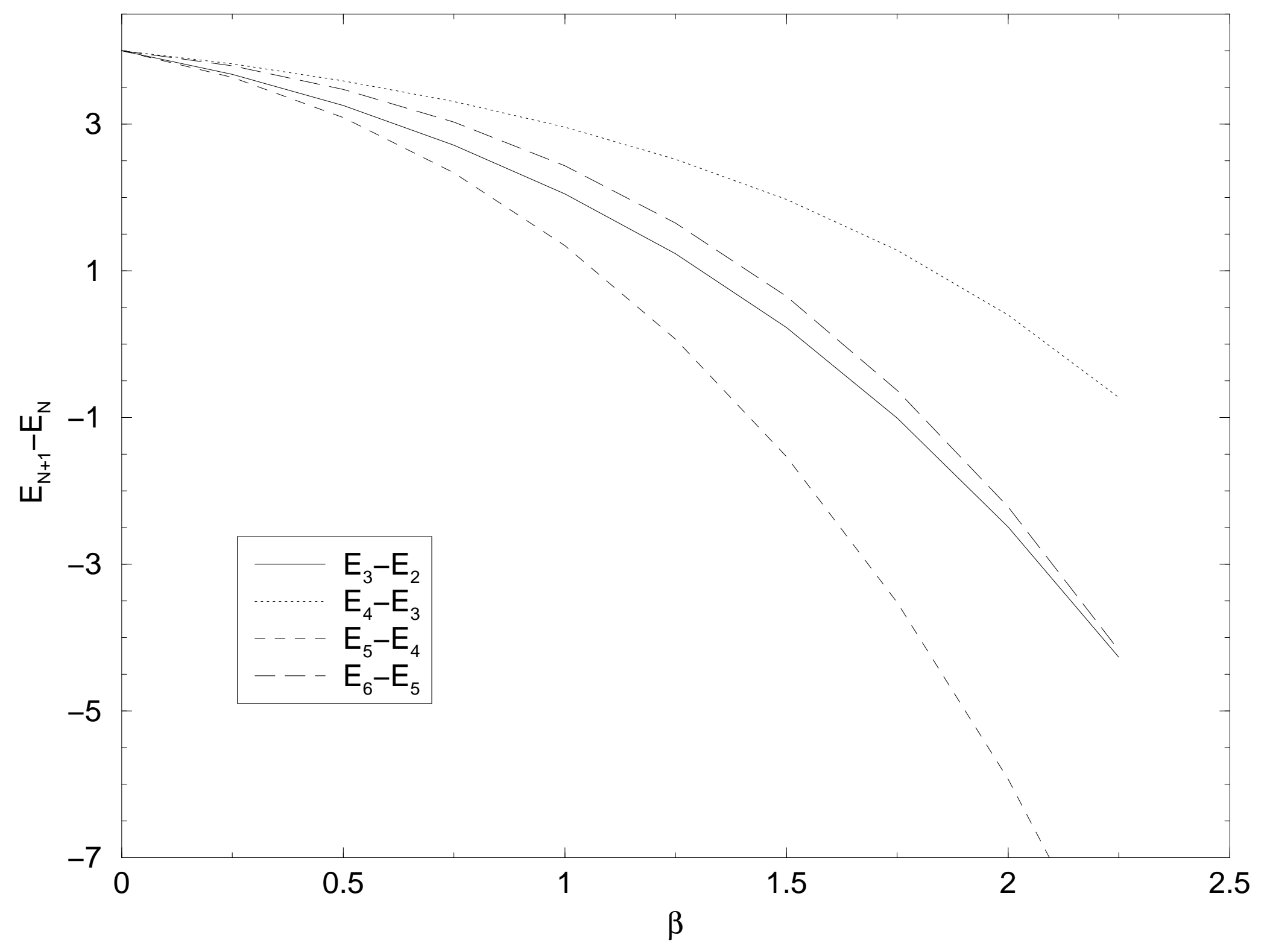




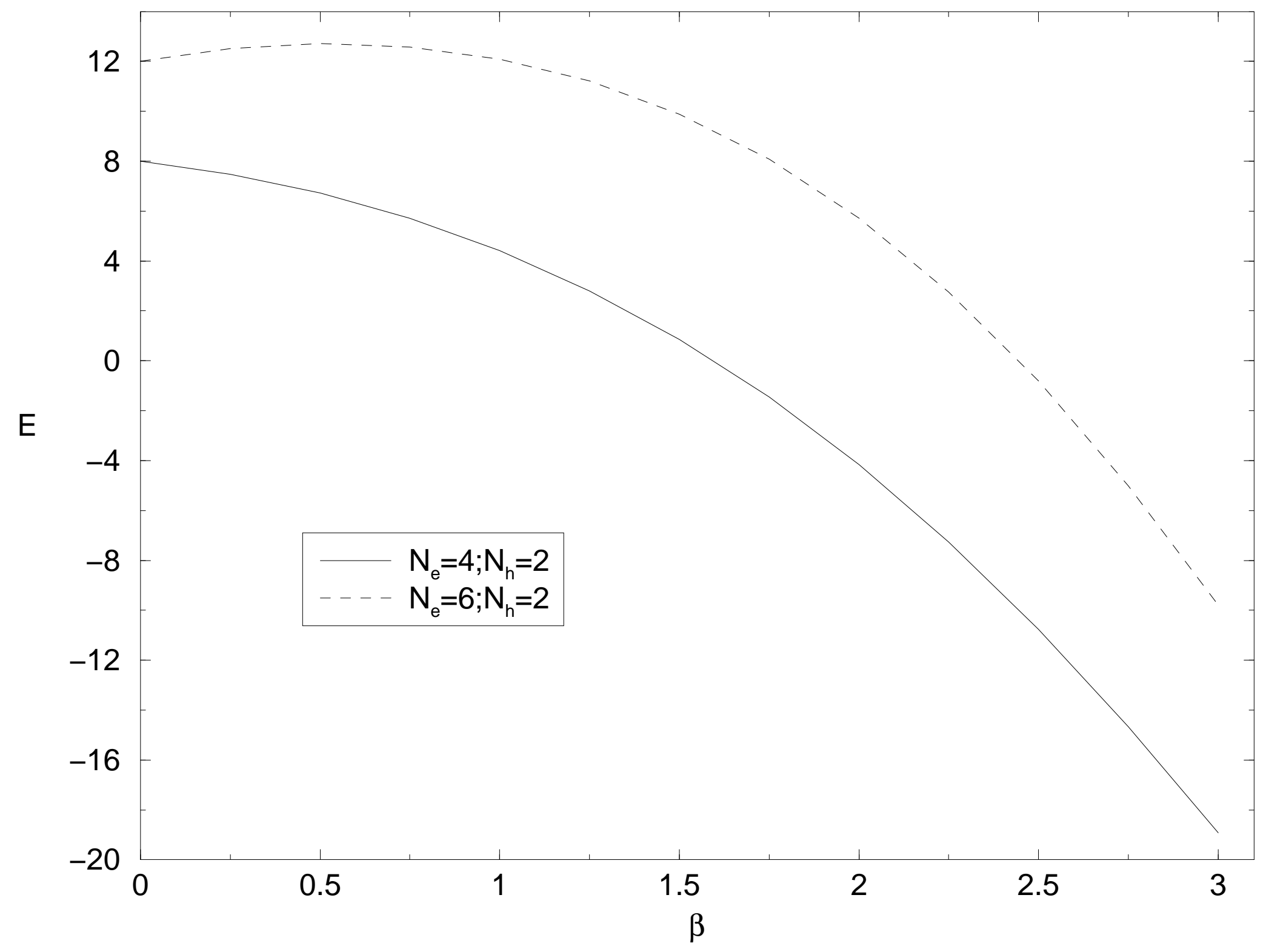

